\title{
Experiencia de voluntarios universitarios nicaragüenses: de la mano con la antropología social
}

\author{
Experience of Nicaraguan university volunteers: hand in hand with social anthropology
}

Jossarys Massielle Gazo Robles

Docente e investigadora del Departamento de Antropología

Universidad Nacional Autónoma de Nicaragua

ID Orcid: https://orcid.org/0000-0002-0989-4827

Jmg993@hotmail.com

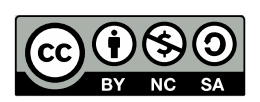

"Enseñar no es transferir conocimiento, sino crear las posibilidades para su propia producción o construcción”.

Paulo Freire

\section{Resumen}

La universidad es una institución social, que debe cumplir con la responsabilidad social universitaria (RSU). En respaldo a lo antes mencionado la Universidad Nacional Autónoma de Nicaragua (UNAN-Managua), como una de las instituciones de educación superior más grande del país, pertenece a la Red Nacional del Voluntariado, es decir, la instancia participativa, democrática y representativa de las organizaciones voluntarias de Nicaragua. Por consecuente bajo esa misma línea institucional, como elemento motivador, se creó en el 2013 el Voluntariado Social de la carrera de Antropología Social, Facultad de Humanidades y Ciencias Jurídicas, culminando en el 2016, siendo un espacio universitario para demostrar la solidaridad organizada de la intervención del sector educativo, ejecutando acciones en benéfico de la comunidad, así como fortalecer una mayor sensibilidad social y unidad de los estudiantes respecto a las problemáticas y necesidad de los habitantes más vulnerables de la cuidad. Por ende, el objetivo de este estudio es explicar como el voluntariado universitario de la carrera de antropología social, se convirtió en un ámbito de interés para fortalecer los procesos de formación universitaria como expresión de un aprendizaje servicio; y se analizará la acción voluntaria por medio de la responsabilidad e implicación de los estudiantes en las diferentes actividades, las cuales permiten: ampliar sus redes sociales, aumentar su realización personal, agregar valor al currículo, contrastar conocimientos, descubrir competencias, sentirse útil para la sociedad, invertir tiempo libre y dejar huella.

Palabras claves: Voluntariado social, voluntariado universitario, antropología nicaragüense, responsabilidad social universitaria, agentes de cambio. 


\begin{abstract}
The university is a social institution, which must comply with university social responsibility (RSU). In support of the aforementioned, the National Autonomous University of Nicaragua (UNAN-Managua), as one of the largest institutions of higher education in the country, belongs to the National Network of Volunteers, that is, the participative, democratic and representative institution of the voluntary organizations of Nicaragua. Consequently, under the same institutional line, as a motivating element, in 2013 the Social Volunteer of the career of Social Anthropology, Faculty of Humanities and Legal Sciences was created, culminating in 2016, being a university space to demonstrate the organized solidarity of the intervention of the educational sector, executing actions in charity of the community, as well as strengthening a greater social sensitivity and unity of students regarding the problems and need of the most vulnerable inhabitants of the city. Therefore, the objective of this study is to explain how the university volunteering of the career of social anthropology, became an area of interest to strengthen the processes of university education as an expression of service learning; and voluntary action will be analyzed through responsibility and involvement of students in different activities, which allow: expand their social networks, increase their personal performance, add value to the curriculum, contrast knowledge, discover skills, feel useful for the society, invest free time and leave a mark.
\end{abstract}

Keywords: Social volunteering, university volunteering, Nicaraguan anthropology, university social responsibility, agents of change.

\section{Introducción}

En este artículo científico se analizan específicamente los aspectos relacionados con el concepto de voluntariado universitario, como una parte importante del comportamiento socialmente responsable de las universidades, o también llamado la responsabilidad social universitaria. Se explican las experiencias de los estudiantes universitarios dentro del voluntariado social de la carrera de Antropología social.

Para el análisis del concepto del voluntariado, se retomó diferentes elementos teóricos y posturas conceptuales. De esta manera Soler (2007) afirma que el voluntariado es:

Un conjunto de personas que ha adquirido una conciencia solidaria fundamentada en una visión crítica de la realidad y en su derecho como ciudadano, desarrollando actividades de forma altruista y solidaria, basadas en su libre decisión, en un compromiso con el marco organizativo que facilita un proceso formativo adec- uado. La finalidad última de su colaboración es la transformación de la realidad social, con unos ideales que aspiran a crear un mundo más solidario, justo y pacífico. (pág.25)

Esta postura conceptual, plantea el voluntariado como una herramienta para ejercer el derecho a participar y transformar la realidad que nos rodea. Los voluntariados, constituyen la vanguardia que toda sociedad necesita para avanzar hacia un sistema de convivencia y de relaciones sociales que privilegie la cultura de la solidaridad.

Generalmente los voluntarios(as) cumplidos y efectivos son personas ocupadas que organizan su tiempo y asumían responsablemente el cumplimiento de las tareas a que se han comprometido. (Ramírez, 2001)

Es importante explicar las modificaciones que han surgido sobre la esencia del voluntariado. Por ende se realiza una clasificación entre las diferencias del voluntariado tradicional y el actual, que plantea Soler (2007): 
El voluntariado tradicional, su población objetivo (son los pobres y menesterosos). El tipo de intervención (el individuo es un mero receptor de la ayuda). Las consecuencias de la intervención (se manifiesta en la pasividad del usurario). El objetivo de la ayuda (la tarea es un fin en sí misma). La capacitación (con buena voluntad es suficiente). Las motivaciones (religiosos o morales) y el reconocimiento legal (era sin control normativo). Por su parte el voluntariado actual se caracteriza por, su población objetivo (dirigido a cualquier persona miembro de la comunidad). El tipo de intervención (Implicación de la comunidad para resolver sus dificultades). Las consecuencias la intervención (participación activa). El objetivo dela ayuda (Únicamente es un medio para el voluntariado). La capacitación (formación adecuada). Las motivaciones (pluralidad de motivos) y el reconocimiento legal (sujeto a normativa jurídica). (pág.52)

Según las Naciones Unidas (2011) las acciones de voluntariado se caracterizan por tres elementos claves:

1. Voluntad libre (la acción debe llevarse a cabo de forma voluntaria, de acuerdo con la propia voluntad libremente expresada de la persona y no como una obligación impuesta por una ley, un contrato o un requisito académico). 2.Motivación no pecuniaria (La acción no debe levarse a cabo primordialmente para la obtención de una recompensa económica). 3.Beneficio para otros (la acción debe perseguir el bien común $\mathrm{y}$ favorece de forma directa o indirecta a personas ajenas a la familia o el hogar del voluntario o bien realizarse en favor de una determinada causa, incluso si la persona que actúa como voluntaria también resulta beneficiada. (pág.4)

Ahora bien, analizar el voluntariado al interior de las universidades, es preguntarse acerca del voluntariado en la gestión de entornos éticos universitarios, por la concepción implícita de la acción social en los procesos educativos es necesario. Por ende Arias (2008) "afirma que el voluntariado universitario hace alusión no solo al voluntariado que tiene como protagonistas a los jóvenes universitarios, sino también a toda la política universitaria y al conjunto de actividades llevadas a cabo dese la comunidad universitaria orientadas hacia este campo".
Se trata, por lo tanto, de un concepto amplio que abarca y engloba a toda la comunidad universitaria y a todos los ámbitos que como universidad le son propios.

Los procesos de voluntariado otorgan a la universidad la oportunidad de formar a sus estudiantes en valores tan importantes como el respecto, la tolerancia o la empatía con el otro.

Dentro del marco jurídico nicaragüense, en la LEY No. 543, Ley del Voluntariado Social, se plantean que el voluntariado social es: "el conjunto de actividades de interés general, desarrolladas por voluntad propia por personas naturales siempre que las mismas no se realicen en virtud de una relación contractual laboral" (Asamblea Nacional de la Republica de Nicaragua, 2005, pág. 2).

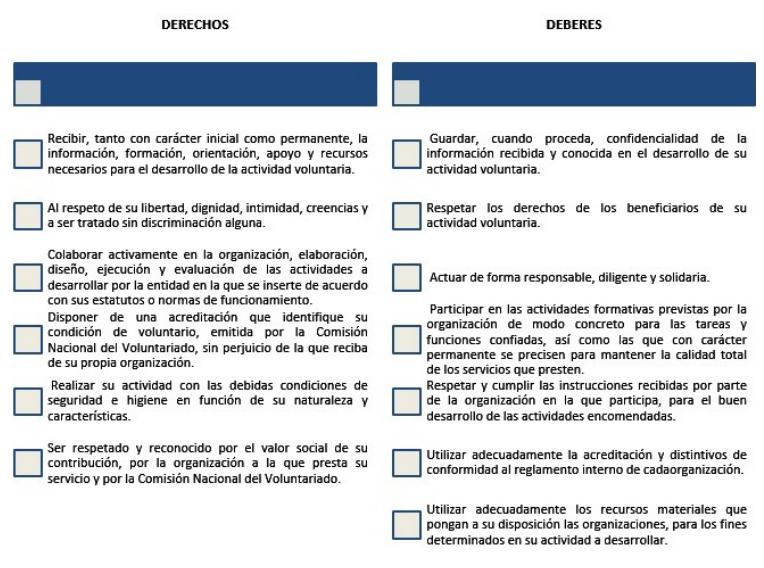

Cuadro 1. Descripción de los derechos y deberes que tienen los voluntarios según la LEY No. 543, Ley del Voluntariado Social.

Por ende, se concibe por voluntarias y voluntarios, las personas naturales que se comprometan libremente a realizar actividades de carácter altruista, solidario y social.

\section{Metodologia}

La investigación se desarrolló desde un corte etnográfico, con una perspectiva del paradigma cualitativo y tipo de estudio explicativo. Se llevó a cabo la revisión bibliográfica, por medio de la ubicación de las fuentes, revisión, selección y adopción de la información referida sobre el voluntariado universitario.

Se utilizó la transliteración para la transcripción de las entrevistas, para desarrollar el análisis e interpretación 
de los datos se recurrió a la teoría fundamentada en los datos, mediante la identificación de códigos, conceptos y categorías. Además, como coordinadora del voluntariado del departamento de Antropología social, durante el 2015-2016, se pudo aplicar la observación participante.

\section{Análisis de los resultados}

El voluntariado social del departamento de Antropología, Facultad de Humanidades y Ciencias Jurídicas, de la Universidad Nacional Autónoma de Nicaragua (UNAN-Managua), era una organización social estudiantil multidisciplinar, con una gran sensibilidad social, que permitía alcanzar diferentes objetivos sociales. Dicho voluntariado, se desarrolló bajo el enfoque de la responsabilidad social, es decir "la capacidad de respuesta que tiene una institución, frente a los efectos positivos o negativos de sus acciones sobre la comunidad y el medio ambiente con los cuales se relaciona" (Grajeda Gonzales, 2010).

Se inició el espacio del voluntariado en el 2012 finalizando en el 2016. Los docentes investigadores de antropología encargados de llevar a cabo el voluntariado social, eran los coordinadores de Extensión y Proyección Social del Departamento de Antropología, fueron: MSc. Javier Rodríguez (Periodo 2012), MSc. Ernesto Gómez Salazar (Periodo 2013-2014) y MSc. Jossarys Gazo Robles (Periodo 2015-2016).

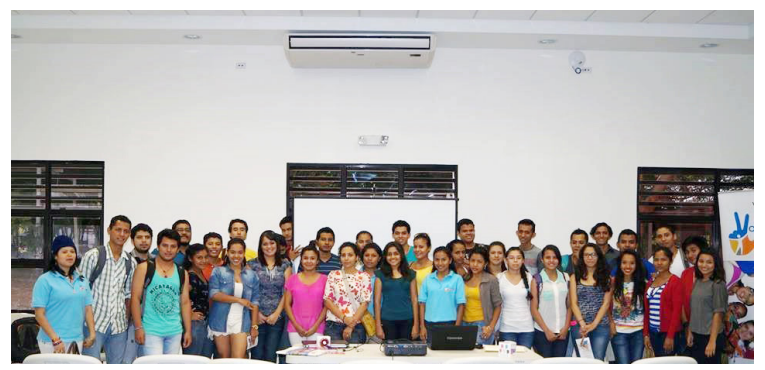

Imagen 1. Voluntarios del departamento de Antropología, conformado por las carreras de: Antropología, Psicología, Comunicación para el desarrollo, Trabajo social y Derecho, participando en los Módulos de Capacitación a los nuevos voluntarios. Dirigido por el Coordinador Nacional del Voluntariado de WORD VISION, MSc José Poveda y por la coordinadora de extensión y proyección social del departamento de Antropología, MSc. Jossarys Gazo.
El voluntariado social de la carrera de Antropología, trabajó con las instituciones/organizaciones: Centro Cultural Batahola Norte, Proyecto Olla, Movimiento Luis Alfonso Velázquez (MILAFV), WORLD VISION y el Programa de Radio Music Box - UNAN. Los ejes que se trabajan estaban encaminados a la prevención de la violencia, niñez, educación, género, derechos humanos, nutrición y conservación de la cultura. Para llevar a cabo las actividades, se realizaba una agenda de trabajo con dichos ejes, se planificaban la visita de acercamiento, reuniones con él o la encargada de la coordinación de extensión y proyección social del departamento de Antropología y con el representante encargado del voluntariado de las instituciones/organizaciones.

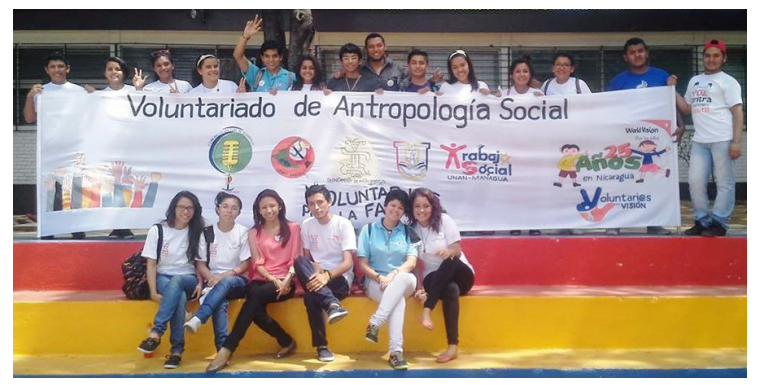

Imagen 2. Parte de los Voluntarios de la carrera de Antropología Social desde 1 año hasta $V$ año. En la manta se plasmaron los logos de las diferentes carreras que hacían parte del voluntariado del Departamento de Antropologia.

Desde el 2012 hasta el 2014, el voluntariado de Antropología se caracterizaba por trabajar solamente con estudiantes de Antropología (desde I año hasta $\mathrm{V}$ año), haciendo un total de 20 estudiantes, pero en el 2015 y 2016, bajo mi coordinación se dio un nuevo rumbo, contando con la participación de estudiantes pertenecientes a otras carreras de la Facultad de Humanidades y Ciencias Jurídicas como: Comunicación para el Desarrollo, Derecho, Psicología y Trabajo Social. Además,

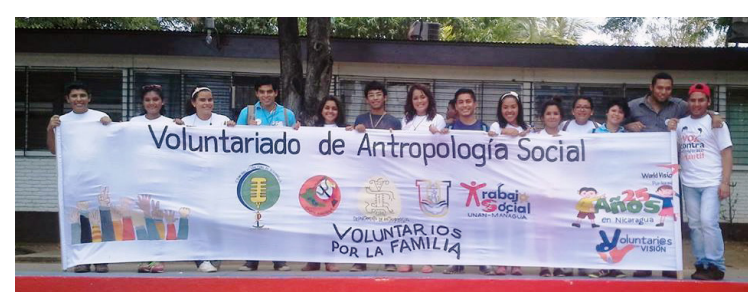

Imagen 3. Parte de los estudiantes de las carreras de Antropología Social y Psicología, perteneciente al voluntario del Departamento de Antropología. 
de la participación de estudiantes que no pertenecían a dicha facultad, como es el caso de la carrera de Medicina, haciendo un total de 60 voluntarios.

Las motivaciones que tenían los estudiantes para participar en el voluntariado social eran: el desarrollo personal y profesional. Los universitarios desarrollaron sus competencias profesionales a través del voluntariado ayudando, por ejemplo, a los niños en las escuelas o personas mayores en los centros de discapacitación.

El estudiante, a nivel personal, aprende a "hacer cosas" que nunca ha hecho, además se enfrenta a nuevos elementos socioculturales, a otras mentalidades, aprendiendo en suma a adaptarse a unas nuevas condiciones de vida. "Y de otra parte, la propia recompensa emocional que supone realizar una actividad de carácter altruista, además el poder trabajar en equipo y conocer nueva gente" (Khasanzyanova, 2011).

Esto es reafirmado por la estudiante activa y destacada del V año de Psicología, Anameyling Guevara, miembro del voluntariado social del departamento de Antropología en el 2015-2016.

Me pareció una fructífera experiencia. Aprendí bastante tanto de mí, como del proyecto. El haber estado en el voluntariado me ayudó para desarrollar mis habilidades, poder mejorar mi oratoria, realizar el plan a seguir en las sesiones, conocer a profundidad los temas abordados como la comunicación, resolución de conflictos, etc. Fue algo desafiante puesto que la pena debía desaparecer para poder hacer una correcta intervención. (Guevara, 2018)

Por ejemplo, en el 2015, los voluntarios de la carrera de Antropología, recibieron una convocatoria por parte de WORLD VISION, para trabajar de forma temporal como traductores, dentro de una de las campañas para la niñez. Siendo los beneficiados los estudiantes que cumplían los requisitos: ser voluntariado activo de la carrera de Antropología, haber participado en las campañas de WORLD VISION, ser una persona dinámica y responsable con las asignaciones, contar con flexibilidad de tiempo sin afectar sus horarios de clases en la universidad y tener dominio del idioma de Ingles.
La acción del voluntariado de la carrera de Antropología, cumplió con los requisitos que indica la LEY No. 543, Ley del Voluntariado Social:

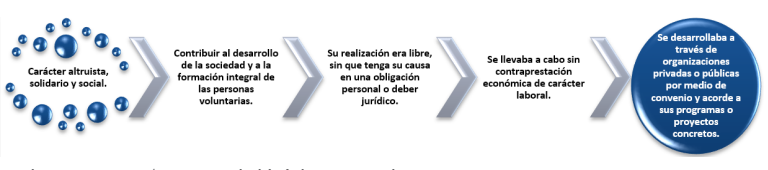

Imagen 5. Acción del Voluntariado

A nivel de gestión, cada semestre, se realizaba la divulgación de la convocatoria para el nuevo ingreso al Voluntariado Social, al finalizar ese periodo, se abrían las inscripciones. Luego se realizaba un taller de implementación de plan de inducción a los nuevos voluntarios. Más adelante se llevaba a cabo una reunión de bienvenida a todos los voluntarios. Se realizaba reunión con la coordinación y planeación de la visita de campo con voluntarios. En otro espacio se desarrollaba las capacitaciones modulares con voluntarios, es decir un curso de formación propia, en muchos casos con metodología elaborada a partir de la experiencia de la propia entidad. Así, el futuro voluntario tiene dos enfoques para recibir una buena formación: por parte de la Universidad y por parte de la institución/organización.

Las capacitaciones brindadas también me ayudaron a desarrollarme, tenía dominio de lo elemental del proyecto, supe que uno debía hacerse parte de la misión, visión, valores, deberes y derechos para poder crear un impacto. También desarrollé mi liderazgo, y lo más importante me encantó convivir con la gente, conocer otros puntos de vista y poder ayudar en las necesidades de las personas. (Guevara, 2018)

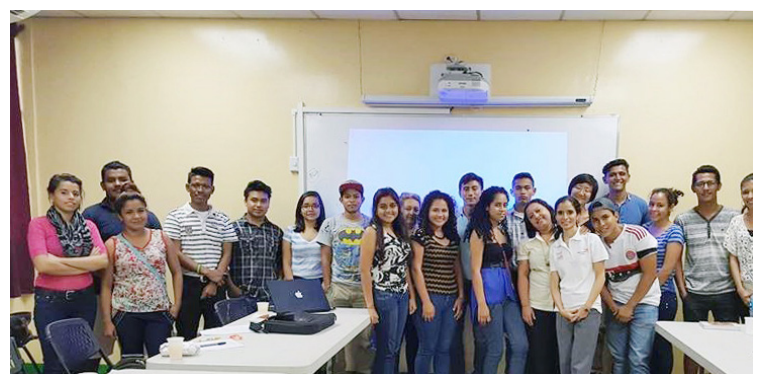

Imagen 3. Parte de los estudiantes de las carreras de Antropología Social y Psicología, perteneciente al voluntario del Departamento de Antropología. 
Por último, se realizaba la actividad de reconocimiento a voluntarios, en el marco de la celebración del día Nacional del Voluntario Nicaragüense, el cual se desarrollaba el 5 de diciembre, esta actividad era planificada por WORLD VISION.

Por ejemplo, en el marco de la celebración del este día Internacional del Voluntario, en el 2015, WORLD VISION reconoció la labor de voluntarios y voluntarias de la Universidad Politécnica de Nicaragua y los estudiantes de psicología y Antropología que pertenecían al voluntariado de la carrera de Antropología de la Universidad Nacional Autónoma de Nicaragua - Managua quienes por más de tres meses apoyaron la iniciativa «Tienda que cambia vidas» durante la campaña Quiero Leer para Aprender.

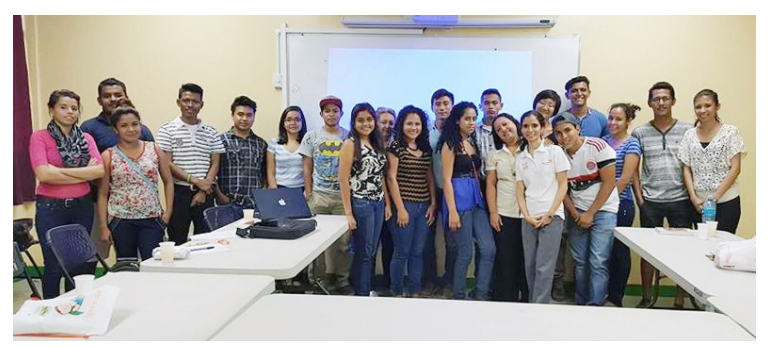

Imagen 6. Parte de los Voluntarios del departamento de Antropología (Estudiantes de Antropología, Trabajo Social, Psicología, Comunicación para el Desarrollo), la coordinadora del Voluntariado de WORD VISION en el 2017, MSc. Miriam Díaz y la coordinadora de Extensión y proyección social del departamento de Antropóloga y encargada del voluntariado, Msc. Jossarys Gazo

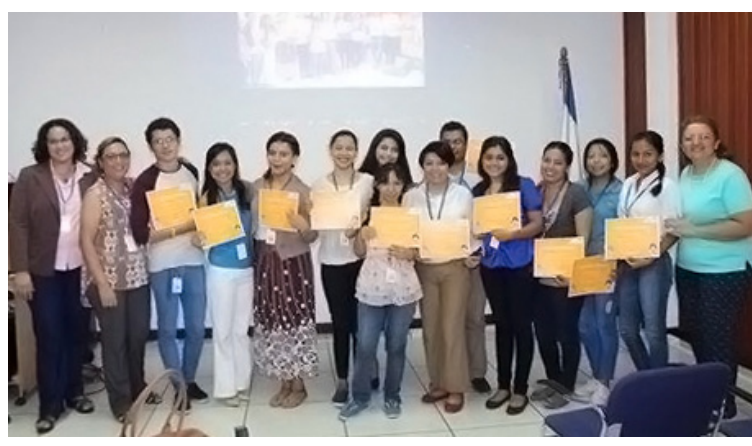

Imagen 7. Reconocimiento por parte del WORD VISION, por sus destacadas participaciones en el voluntariado. Ángeles Cerda, estudiante de la carrera de Antropología y Anameyling Guevarra estudiante de Psicología.
En el 2016, como parte de extensión y proyección social, se realizó actividad con WORLD VISION, conformado por nuevos y antiguos miembros, de diferentes carreras como: Antropología, Comunicación para el desarrollo, Psicología y Trabajo Social. Actividad llevada a cabo por: Lic. Jossarys Gazo Coordinadora de Extensión y Proyección de Antropología. MSc. Miriam Díaz. Coordinadora Nacional de Voluntariado de WORLD VISION, MSc. Karen Gonzales. Coordinadora de Protección de WORLD VISION.

La actividad consistía en presentar la agenda de trabajo anual planificada para llevar a cabo las actividades donde los estudiantes desde sus diferentes carreras podían aportar para la causa.

Más adelante se llevó a cabo la actividad con el PDA «Aguas Azules» comunidad las Playitas en las Isletas de Granada. Participantes: MSc. Jossarys Gazo, Coordinadora de extensión y proyección social de la antropología social y Lic. José Poveda, Representante de WORLD VISION Nicaragua, Representantes del PDA y los Promotores de Salud. Estudiantes de las carreras de Antropología, Trabajo Social, Psicología, Trabajo social, Comunicación para el Desarrollo y medicina. Se visitó la escuela primaria, donde se compartió con los niños, maestros y padres de la escuela, por medio de dinámicas populares, enseñanza del lavado de manos y dientes a los niños, y el uso adecuado de alimentos en base a soya. Se les llevo piñatas y juguetes a los niños.

Otra activada fue la participación en la Tienda que cambia vidas, se encontraban en los diferentes centros comerciales. Las voluntarias de la carrera de Antropología de la UNAN-Managua y el equipo de WORLD VISION, apoyaban a la involucración de más personas en el Programa Quiero Leer para Aprender, el objetivo era Educar con Ternura.

Participación en la Gran Recolecta Nacional "Juntos por Haiti", por parte de WORLD VISION

Se apoyó la Gran Recolecta de los niños y niñas del MILAFV, en coordinación con el Voluntariado de Antropología Social y el apoyo de UNEN, el cual brindo el aporte alimenticio. 


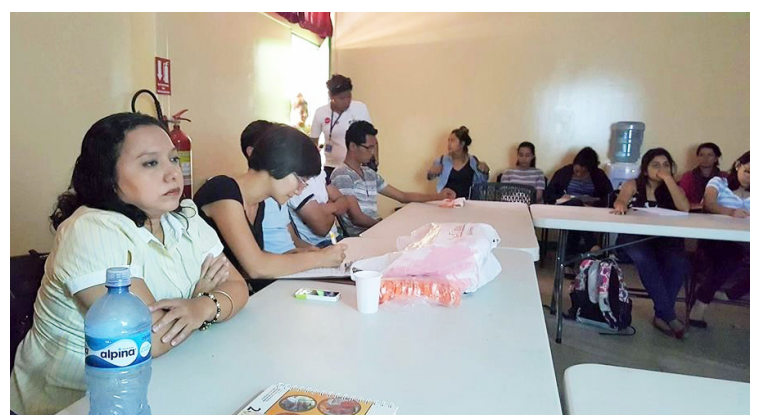

Imagen 9. Reunión de Presentación del plan de trabajo y nueva campaña de niñez en la que participarían los voluntarios.

En el caso de PROYECTO CEB, los voluntarios brindaban talleres con metodología popular, a los miembros de familia del proyecto, bajo los temas de educación en valores, conflictos, ¿Cómo mejorar la autoestima?

Cada una de las acciones voluntarias desarrolladas eran iniciativas desinteresadas que nacían del interés de las instituciones y de los futuros profesionales universitarias para contribuir a mejorar la comunidad, colocando al servicio de la misma aquellas capacidades y conocimientos adquiríos en las aulas universitarias. Existía un impulso ético y social de los estuantes que los movía siendo además una interesante instancia para desarrollar valores socialmente responsables y de liderazgo social.

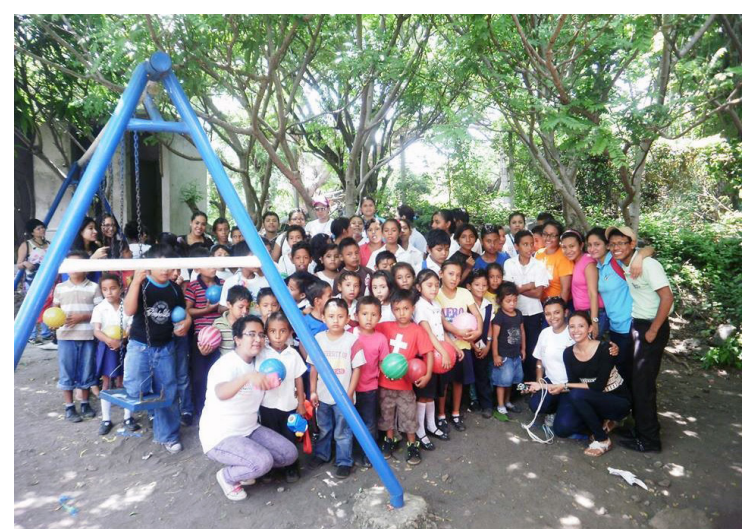

Imagen 10. Niños beneficiados con actividades que desarrollaron los voluntarios del departamento de Antropología junto a WORLD VISION.

\section{Discusión de resultados}

El voluntariado de la carrera de Antropología, que se conformaba por estudiantes de las carreras de Antropología,
Comunicación para el Desarrollo, Derecho, Psicología y Trabajo Social, en la práctica se apegaba a las características del voluntariado actual que plantea Soler (2007), es decir la población objetivo se caracterizaba por ser beneficiada cualquier persona miembro de la comunidad, que las instituciones/ organizaciones seleccionaran. El tipo

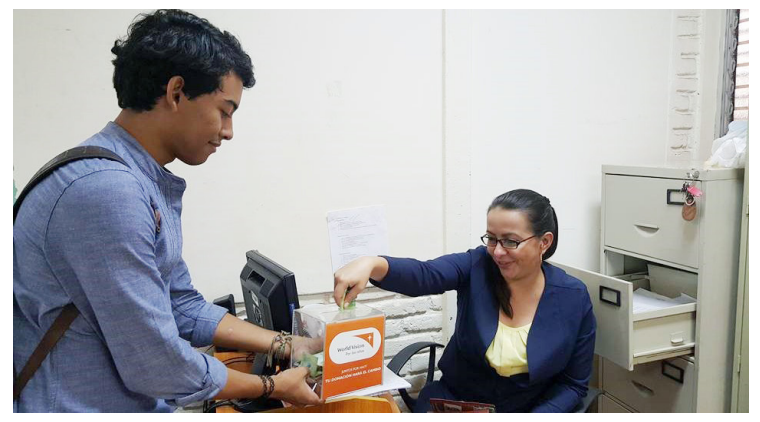

Imagen 11. Trabajadora de la UNAN Managua, colaborando en el gran recolecta nacional de WORLD VISION, en beneficio de la Niñez.

de intervención, iba del mando con la implicación de la comunidad para resolver sus dificultades. Las consecuencias la intervención se manifestaba en una participación activa. El objetivo de la ayuda únicamente era un medio para el voluntariado. Las motivaciones de los voluntariados eran pluralidad de motivos y el reconocimiento legal estaba sujeto a normativa jurídica. La capacitación de los voluntariados se daba por medio de una formación adecuada, eran un total de 4 módulos.

La modalidad que el voluntario del departamento de Antropología asumía en el ámbito universitario era el formal, es decir a través de la denominada proyección social que la universidad realiza como

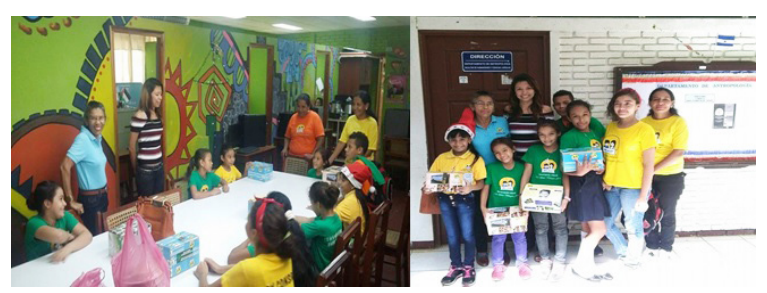

Imagen 13. Niños del MILAFV en la Recolecta Nacional para seguir con sus programas de beneficencia, acompañados de la compañera Olga Artiles Jarquín Avendaño, coordinadora en la capital del Movimiento Infantil Luis Alfonso Velázquez Flores (MILAVF) y la coordinadora del voluntariado del departamen- 
institución. Estaba orientado a que los voluntarios aplicaran los conocimientos y habilidades adquiridas más allá de una concepción asistencial. Se vinculaba con instituciones que trabajan con voluntarios que les interesaba trabajar con estudiantes.

Los voluntarios realizaban las actividades de manera proactiva y totalmente por motivación propia, no se les daba incentivos económicos ni era parte de requisitos para obtener o poder mantener becas estudiantiles, sino que eran personas que realizaban las actividades para sentirse "socialmente útil" como forma de retribuir a la sociedad los conocimientos y experiencias adquiridos a través del tiempo o también para invertir su tiempo.

Lo anteriormente mencionado, lo explica Marcel Mauss y Branisslow Malinoski, los cuales establecieron que la donación presume un sentido de obligación y reciprocidad, un sentimiento de inclusión. La concepción del don ha sido tomada para subrayar la gratuidad de las relaciones de colaboración y controvertir la lógica utilitarista.

Las actividades de voluntariado del departamento de Antropología (Antropología, Comunicación para el desarrollo, Derecho, Psicología, Trabajo Social), les permitió fortalecer su sensibilidad social, aun cuando formalmente no integran las mallas curriculares ni son expresión de alguna estrategia de enseñanza aprendizaje utilizada por los docentes

El impacto del voluntariado se manifiesto en dos ámbitos el de proyección y el del RSU. El primer impacto fue al proyectar la carrera de Antropología social más allá del ámbito del recinto universitario. El segundo impacto de la Responsabilidad Social Universitaria (RSU), se manifiesto cuando los estudiantes ponen en servicio de la comunidad sus conocimientos y capacidades para atender alguna necesidad social configurando procesos de aprendizaje servicio no generados desde el plan curricular, y finalmente favorece el desarrollo de iniciativas que se proyectan en el tiempo más allá del ámbito universitario para propiciar un cambio social que permita un desarrollo más sustentable y justo en la sociedad

En fin, la experiencia de voluntariado de la carrerea de Antropología, aportó en la promoción personal, profesional y formativa que será incluida en su currículum personal.
La experiencia del voluntariado de la carrera de Antropología, es un antecedente que permite analizar el verdadero desafío de la Universidad Nacional Autónoma de Nicaragua (UNAN) como universidad socialmente responsable respecto el voluntariado universitario, radica no solo en elegir qué tipo de voluntariado debe promover desde su interior, sino que muy especialmente ser capaces de articularlo con la formación y la investigación, para lograr que los voluntarios universitarios sean ciudadanos plenamente consiente respecto de las razones por las cuales se originan los problemas sociales, para intentar incidir en la búsqueda de soluciones definitivas que permitan superar dichas desigualdades dentro de la sociedad. Es necesario transformar las actividades de voluntariado universitario en un aspecto integral del quehacer universitario y no como un hecho aislado o circunstancial.

Se recomienda que se permita que los estudiantes que solicitan una acreditación del trabajo realizado se le acredite legalmente esta experiencia "profesional" de voluntario.

El campo universitario UNAN Managua al articular el voluntariado social con la formación, se convertirá en un instrumento educativo y formativo que contempla la formación que los estudiantes reciben en las aulas. Los años de estudio universitarios, se convierten por ende para los jóvenes en años de aprendizaje social, durante los cuales se fomentan la actuación del universitario en la transformación de la sociedad.

Con lo dicho anteriormente se fomentaría y apoyaría la investigación destinada a: identificar las necesidades de acción voluntaria y de las comunidades en el entorno de las universidades; y conocer la situación del voluntariado en la Universidad y en el Estado, el impacto de la acción voluntaria y las necesidades formativas y de acción de voluntarios.

\section{Conclusión}

El voluntariado universitario nicaragüense requiere de una nueva y correcta dirección y aprehensión, que logre resignificar el impacto de la relación Universidad-Sociedad. Fue precisamente en este 2018, en Nicaragua, donde esta necesidad imperante de discusión se dio a través del III Encuentro de Voluntariado Universitario, siendo la UNAN Managua, la institución anfitriona. 
Se debe cambiar la perspectiva del voluntario tradicional, desde una perspectiva de desarrollo cultural, concibiéndolo como un voluntario cultural como un promotor de la participación ciudadana para la transformación social a través de herramientas orientadas al cultivo de la creatividad, la divulgación del conocimiento, la difusión del arte, la valoración social y económica del patrimonio. El voluntario cultural se convierte en un agente de cambio en la medida que promueve y alienta la participación, moviliza personas, recursos humanos, técnicos y financieros para hacer de las manifestaciones culturales un recurso para el desarrollo.

Es un reto a futuro formar voluntarios en los procesos de desarrollo cultural parte del reconocimiento del voluntariado como la posibilidad de presencia social y participación en tareas políticas, de planificación, innovación tecnológica, culturales, sin las particularidades de una dinámica profesional, pero con estándares de responsabilidad y trabajo bien realizado.

El voluntario social, debería ser incluida como una estrategia de enseñanza de formación universitaria que ayude a romper con la educación bancaria, que los estudiantes dejen de ser estudiantes pasivos de su propio proceso de formación donde predomina la clase de los docentes universitarios de tipo expositiva, teórica y absolutamente desconectada del entorno.

Un último desafió seria que la UNAN mangua, logre articular el voluntariado universitario con el emprendimiento social como parte de la Responsabilidad social Universitaria (RSU). Es decir que las acciones del voluntariado universitario que se hagan de manera interna, logren transformarse en verdaderos emprendimientos sociales.

\section{Bibliografía}

Arias, S. (2008). Voluntariado Universitario. Guia para su gestion en las universidades madrileñas. Madrid: Direccion General de Voluntariado y Promocion Social.

Asamblea Nacional de la Republica de Nicaragua. (22 de Junio de 2005). LEY No. 543 Ley del voluntariado social. Managua, Nicaragua: Asamblea Nacional de la Republica de Nicaragua.

Grajeda Gonzales, L. (2010). De la Extension y Proyeccion social hacia la Responsabilidad Social Universitaria. Argentina.

Guevara, A. (1 de Diciembre de 2018). Experencia en el Voluntariado del Departamento de Antropologia. (J. Gazo, Entrevistador)

Khasanzyanova, A. (2011). La formación para el voluntariado en la Universidad de Granada (Vol. 2). Journal for Educators, Teachers and Trainers, Vol. 2.

Naciones Unidas. (2011). V Informe sobre el estado del voluntariado en el mundo. Valores universales para alcanzar el bienestar mundial. Naciones Unidas.

Ramírez, V. B. (2001). Referencias del Consejo de Comunidades Solidarias . Colombia: Dra. Mónica Carrillón.

Soler, P. (2007). Factores psicosociales explicativos del voluntariado unversitario. Tesis Doctoral. España: Universidad de Alicante.

\section{Jossarys Massiel Gazo Robles}

Máster en Métodos de Investigación Científica. Licenciada en Antropología Social. Recibe el Reconocimiento de Excelencia en Investigación a nivel de la Unan-Managua en el 2011. Con el proyecto de innovación ReutilAgua, es galardonada en 2012 por la Grand Valley University, Tager Studio y la Unan. Actualmente se desempeña como docente del departamento de Antropología, donde también coordina el área de Investigación del Departamento de Antropología. Ha participado como tutora en la Jornada de Desarrollo Científico, JUDC 2015-2017. Sus áreas de interés se relacionan con la etnoecología, la medicina tradicional y el rescate de los saberes ancestrales. Ha realizado investigaciones y publicados artículos científicos sobre medio ambiente, plantas medicinales y desarrollo comunitario, desde el enfoque antropológico. Sobre estas temáticas ha liderado también procesos participativos en diferentes comunidades indígenas de Nicaragua. 